\title{
Subarachnoid Hyperattenuation on Flat Panel Detector-Based Conebeam CT Immediately after Uneventful Coil Embolization of Unruptured Intracranial Aneurysms
}

\author{
Y. Shinohara, M. Sakamoto, H. Takeuchi, T. Uno, T. Watanabe, T. Kaminou, and T. Ogawa
}

\begin{abstract}
BACKGROUND AND PURPOSE: Flat panel detector-based CBCT can provide CT-like images of the brain without transferring patients from the angiography suite to a conventional CT facility. Conventional brain CT after uneventful endovascular treatment sometimes shows focal subarachnoid hyperattenuation with contrast leakage, mimicking SAH. Differentiating this finding from SAH is important for immediate postprocedural medical management. We investigated $C B C T$ for detecting subarachnoid hyperattenuation immediately after coil embolization of unruptured cerebral aneurysms.
\end{abstract}

MATERIALS AND METHODS: Thirty-six patients with unruptured cerebral aneurysms undergoing CBCT immediately after uncomplicated coil embolization were included. The relationship between the presence of subarachnoid hyperattenuation and total volume of contrast medium injected, aneurysm size and location, and balloon and stent assistance during embolization was investigated. Statistical analyses were performed with the $\chi^{2}$ test $(P<.05)$.

RESULTS: Nine of the 36 patients (25.0\%) showed focal subarachnoid hyperattenuation within the relevant parent artery territory harboring the aneurysm. Subarachnoid hyperattenuation locations included the ipsilateral superior frontal sulcus $(n=5)$, the bilateral superior frontal sulcus $(n=1)$, and the ipsilateral superior frontal and precentral sulci $(n=3)$. Statistically significant differences were observed between the presence of a subarachnoid hyperattenuation and the total volume of contrast medium injected $(P<.001)$ and aneurysm size $(P<.05)$.

CONCLUSIONS: Subarachnoid hyperattenuation can be detected by CBCT immediately after coil embolization for unruptured aneurysms. The increased amounts of contrast medium to be given before $\mathrm{CBCT}$ and the specific location of the hyperattenuation may help differentiate benign subarachnoid contrast leakage from SAH.

ABBREVIATIONS: $\mathrm{ACA}=$ anterior cerebral artery; $\mathrm{AcomA}=$ anterior communicating artery; $\mathrm{CBCT}=$ conebeam $\mathrm{CT} ; \mathrm{FPD}=$ flat panel detector; $\mathrm{VB}=$ vertebrobasilar artery

A dvances in FPD-equipped angiographic technology have resulted in its increasing use for neuroendovascular treatment. FPD-based CBCT is a 3D imaging tool for the reconstruction of projection data with a rotational C-arm with an FPD. With this technique, CT-like images of the brain parenchyma can be obtained without transferring patients from the angiography suite to a conventional CT facility. ${ }^{1-7}$ Therefore, unpredictable complications, such as intracerebral hematoma and SAH, can be rapidly

Received March 29, 2012; accepted after revision May 31.

From the Division of Radiology (Y.S., T.K., T.O.), Department of Pathophysiological Therapeutic Science, and Division of Neurosurgery (M.S., T.U., T.W.), Department of Brain and Neurosciences, Faculty of Medicine, Tottori University, Yonago, Japan; and Department of Neurosurgery (H.T.), Nojima Hospital, Kurayoshi, Japan.

Address correspondence to: Yuki Shinohara, MD, Division of Radiology, Department of Pathophysiological Therapeutic Science, Faculty of Medicine, Tottori University, 36-1 Nishi-cho, Yonago 683-8504, Japan; e-mail: shino-y@olive.plala.or.jp

http://dx.doi.org/10.3174/ajnr.A3243

detected with CBCT during or immediately after a neuroendovascular procedure. ${ }^{4-6}$

Previous reports suggest that conventional noncontrast CT of the brain sometimes shows subarachnoid hyperattenuation that mimics SAH after uneventful endovascular treatment for cerebral aneurysms. This subarachnoid hyperattenuation is presumably due to the transient impairment of the CSF barrier or a change in vascular permeability by nonionic iodinated contrast medium. ${ }^{8-12}$ Although this radiologic finding is a clinically benign one after neuroendovascular procedures, it is important to differentiate it from $\mathrm{SAH}$, which requires significant immediate postprocedural medical management, including the use of anticoagulants and antiplatelet agents.

There are relatively few systematic evaluations of subarachnoid hyperattenuation on conventional CT following uncomplicated coil embolization of unruptured intracranial aneurysms. ${ }^{10-12}$ Moreover, as far as we know, there are no reports 
describing subarachnoid hyperattenuation on postprocedural CBCT. Although contrast resolution of CBCT is still considered inferior to that in conventional $\mathrm{CT}$, current $\mathrm{CBCT}$ provides an almost CT-like contrast resolution, allowing differentiation between gray and white matter and detection of ventricles and intracerebral hemorrhages. ${ }^{5,13}$ The goal of this study was to investigate the ability of CBCT to detect subarachnoid hyperattenuation immediately after coil embolization of unruptured intracranial aneurysms.

\section{MATERIALS AND METHODS \\ Patients}

This retrospective study analyzed data obtained from all patients with unruptured intracranial aneurysms admitted to our institution between May 1, 2010, and March 23, 2012. Patients with uncomplicated endovascular coil embolization for an unruptured intracranial aneurysm who underwent FPD-based CBCT immediately after embolization were eligible if they had no acute cerebrovascular disease, including intracerebral hematoma, SAH, or infarction on pretreatment CT or MR images and if they underwent intracranial MR imaging, including DWI, T2* -weighted imaging, and FLAIR imaging, within 1 week after treatment. Our study was approved by our institutional review board, and informed consent for endovascular treatment and imaging examinations was obtained from each patient.

\section{Endovascular Procedures}

Endovascular procedures were performed with the patient under general anesthesia. All patients were given intravenous heparin for systemic anticoagulation to maintain an activated clotting time at twice the normal value during catheterization and coil placement. A $5 \mathrm{~F}$ or $6 \mathrm{~F}$ guiding catheter was placed in the internal carotid or vertebral artery via a unilateral or bilateral transfemoral approach. HyperGlide (ev3, Irvine, California) and HyperForm (ev3) balloons were used for balloon-assisted procedures, and intracranial stents (Enterprise Vascular Reconstruction Device; Codman Neurovascular, Miami Lakes, Florida) were used for stent-assisted procedures. Nonionic water-soluble iodinated contrast medium (iohexol, Omnipaque 300; Daiichi-Sankyo, Tokyo, Japan) was used. After embolization, conventional 2D DSA and $3 \mathrm{D}$ rotational DSA were performed to check the status of the treated aneurysm and parent artery.

\section{Imaging Examinations}

CBCT was performed by using a biplane FPD angiography system with 3D rotational capability (Allura Xper 20/10; Philips Healthcare, Best, the Netherlands or Axiom Artis dBA; Siemens, Erlangen, Germany) after 3D rotational DSA. We did not give additional contrast medium to perform CBCT. CBCT data were acquired by using the following parameters: Allura Xper 20/10: acquisition time, 20 seconds; tube voltage, $120 \mathrm{kV}$; projection matrix, $1024 \times 1024$; rotation angle, $210^{\circ}$; total frames, 620 ; dose, $0.9 \mu \mathrm{Gy} /$ pulse; Axiom Artis dBA: acquisition time, 20 seconds; tube voltage, $70 \mathrm{kV}$; projection matrix, $1024 \times 1024$; rotation angle, $217^{\circ}$; total frames, 543 ; dose, $1.2 \mu \mathrm{Gy} /$ pulse. Postprocessing of the CBCT dataset was performed on a commercially available dedicated workstation (XtraVision; Philips Healthcare or
Relationship between each parameter and subarachnoid hyperattenuation on FPD-based CBCT immediately after coil embolization

\begin{tabular}{|c|c|c|c|}
\hline \multirow[b]{2}{*}{ Parameter } & \multicolumn{2}{|c|}{$\begin{array}{c}\text { Subarachnoid } \\
\text { Hyperattenuation }\end{array}$} & \multirow{2}{*}{$\begin{array}{c}P \\
\text { Value }\end{array}$} \\
\hline & + & - & \\
\hline \multicolumn{4}{|l|}{ Location of aneurysm } \\
\hline ICA & 4 & 19 & .0728 \\
\hline MCA & 0 & 2 & \\
\hline AcomA & 3 & 3 & \\
\hline $\mathrm{ACA}$ & 2 & 0 & \\
\hline VB & 0 & 3 & \\
\hline \multicolumn{4}{|l|}{ Size of aneurysm (mm) } \\
\hline$<5$ & 0 & 12 & $<.05$ \\
\hline $5-10$ & 4 & 13 & \\
\hline $10-15$ & 1 & 0 & \\
\hline$\geq 15$ & 4 & 2 & \\
\hline \multicolumn{4}{|c|}{ Amount of contrast medium $(\mathrm{mL})$} \\
\hline$<150$ & 0 & 17 & $<.001$ \\
\hline $150-250$ & 4 & 8 & \\
\hline$\geq 250$ & 5 & 2 & \\
\hline \multicolumn{4}{|l|}{ Balloon assistance } \\
\hline Performed & 3 & 12 & .567 \\
\hline Not performed & 6 & 15 & \\
\hline \multicolumn{4}{|l|}{ Stent assistance } \\
\hline Performed & 4 & 5 & .150 \\
\hline Not performed & 5 & 22 & \\
\hline
\end{tabular}

$\mathrm{X}$-Leonardo; Siemens). Postprocessing resulted in a volume dataset with a $0.98-\mathrm{mm}$ thickness in a $256 \times 256$ matrix format for XtraVision and a $0.47-\mathrm{mm}$ thickness in a $512 \times 512$ matrix format for X-Leonardo. Reconstructions were performed by using the soft-tissue algorithm for XtraVision and kernel-type Hounsfield units with image-characteristics "normal" for X-Leonardo. The dataset was then further processed as multiplanar reconstructions with 5.0-mm section thicknesses. Images were viewed in axial and coronal orientations.

Follow-up MR imaging examinations were performed within 1 week after endovascular therapy by using 3T (Signa Excite HD; GE Healthcare, Milwaukee, Wisconsin). The following sequences were included in the protocol of these studies: DWI: TR/TE/excitation, $6000 / 65.6-71 \mathrm{~ms} / 1$; flip angle, $90^{\circ}$; section thickness/intersection gap, 5/1.5 mm; FOV, $21 \mathrm{~cm}$; matrix size, $512 \times 512$; two b-values of 0 and $1000 \mathrm{~s} / \mathrm{mm}^{2}$; T2*-weighted imaging: TR/TE/ excitation, 750-850/20-30 ms/1; section thickness/intersection gap, 5/1.5 mm; FOV, $23 \mathrm{~cm}$; matrix size, $512 \times 512$; FLAIR imaging: TR/TE, 10,002/116 ms; section thickness/intersection gap, 5/1.5 mm; FOV, $21 \mathrm{~cm}$; matrix size, $512 \times 512$.

\section{Data Analysis}

CBCT images obtained immediately after embolization were evaluated for any abnormal findings associated with the procedures, such as SAH, intracerebral hematoma, and large infarctions. Subarachnoid hyperattenuation was regarded as the presence of focal hyperattenuation in the sulci according to the territory of the parent artery harboring the aneurysm and no evidence of SAH on follow-up MR images. Images of all patients were retrospectively reviewed by 2 neuroradiologists (Y.S. and T.O.), who reached consensus regarding image interpretation. The neuroradiologists were not blinded to the location of the treated aneurysm in each case. 

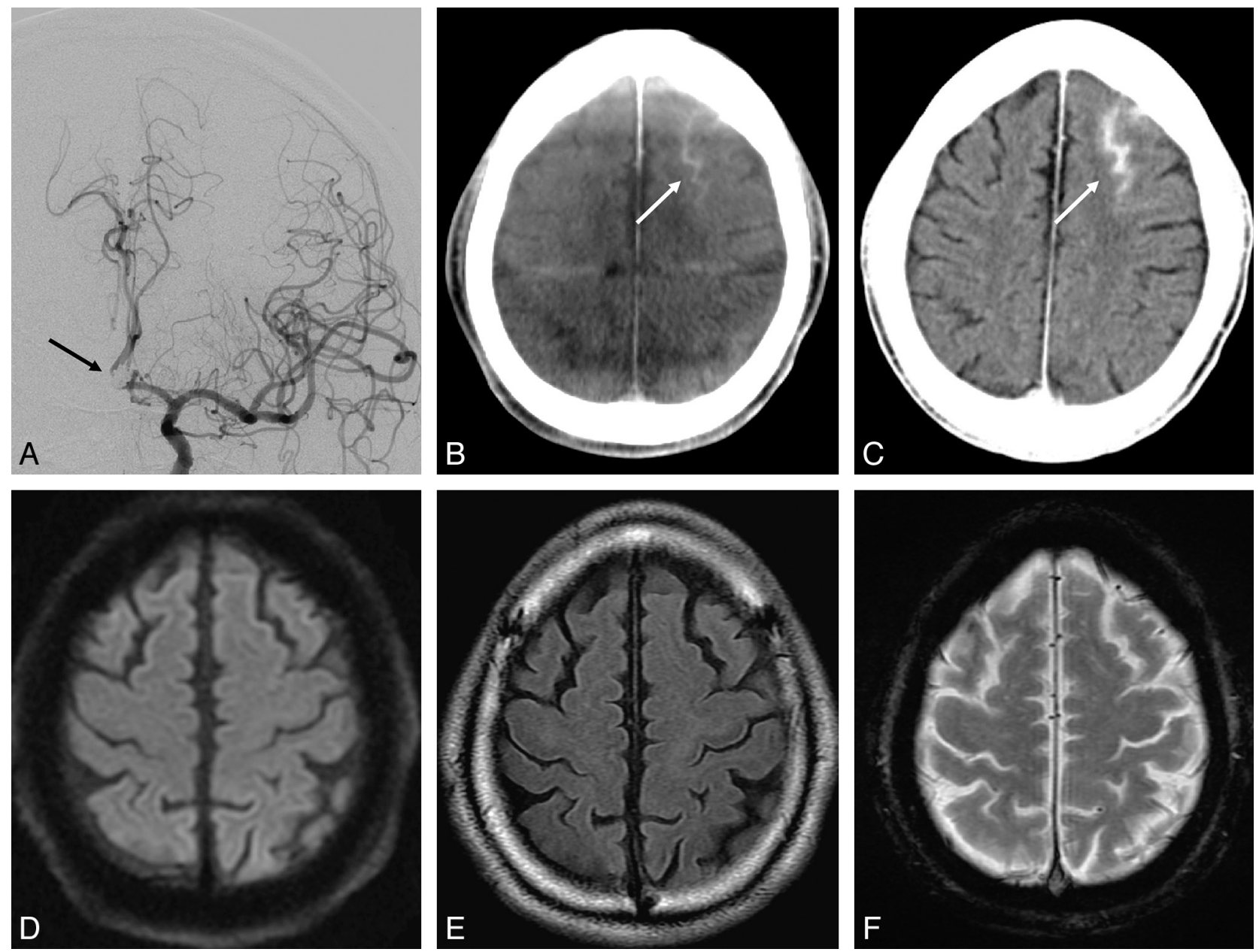

FIG 1. A 63-year-old man with an unruptured 6-mm AcomA complex aneurysm. The patient underwent endovascular embolization for the aneurysm with the guiding catheter in the left ICA. The aneurysm was coiled with balloon assistance. The total volume of contrast medium injected was approximately $300 \mathrm{~mL}$. A, DSA after embolization reveals coil mesh in the left Al-A2 bifurcation (black arrow). B, CBCT immediately after the procedure shows focal subarachnoid hyperattenuation in the left superior frontal sulcus (white arrow). C, Conventional CT 45 minutes after the procedure also shows focal subarachnoid hyperattenuation in the left superior frontal sulcus (white arrow). D, DWI reveals no abnormal high-intensity area indicating acute infarction. E, FLAIR imaging shows no high signal intensity in the sulci indicating SAH. F, T2*weighted imaging demonstrates no low signal intensity in the sulci indicating SAH. These MR images were obtained 111 minutes after the procedure.

In addition, the relationship between subarachnoid hyperattenuation on CBCT and several parameters, including the location and size of the aneurysm, total volume of contrast medium injected, and use of balloon or stent assistance during embolization, was investigated. Aneurysm size was classified into 4 groups: $<5 \mathrm{~mm}, 5-10 \mathrm{~mm}, 10-15 \mathrm{~mm}$, and $\geq 15 \mathrm{~mm}$. The amount of contrast medium was also classified into 3 groups: $<150 \mathrm{~mL}$, $150-250 \mathrm{~mL}$, and $\geq 250 \mathrm{~mL}$. Statistical analyses were performed by using the $\chi^{2}$ test. $P<.05$ was considered significant.

\section{RESULTS}

Thirty-six patients ( 27 women and 9 men; mean age, $62.3 \pm 10.4$ years; range, $41-83$ years) were included in this study. According to the relevant artery territory, the location of the aneurysms was the ICA in 23 patients, MCA in 2 patients, AcomA in 6 patients, ACA in 2 patients (the horizontal segment of the right ACA in 1 patient and the precallosal segment of the right ACA in the other), and the VB in 3 patients. The diameters of the aneurysms were $<5$ $\mathrm{mm}$ in 12 patients, $5-10 \mathrm{~mm}$ in 17 patients, $10-15 \mathrm{~mm}$ in 1 patient, and $\geq 15 \mathrm{~mm}$ in 6 patients. The amounts of contrast me- dium were $<150 \mathrm{~mL}$ in 17 patients, $150-250 \mathrm{~mL}$ in 12 patients, and $\geq 250 \mathrm{~mL}$ in 7 patients. Fifteen patients were treated with balloon assistance, and 9 patients were treated with stent assistance.

On immediate postembolization CBCT, 9 of 36 patients (25.0\%) showed focal subarachnoid hyperattenuation within the relevant parent artery territory harboring the treated aneurysm. The location of the subarachnoid hyperattenuation was the ipsilateral superior frontal sulcus in 5 patients (Fig 1), the bilateral superior frontal sulci in 1 patient (Fig 2), and the ipsilateral superior frontal sulcus and precentral sulcus in 3 patients. Although we could not evaluate the presence of subarachnoid hyperattenuation adjacent to the coiled aneurysm on CBCT due to metallic artifacts, there was no evidence of subarachnoid hyperattenuation or SAH in the basal cistern or Sylvian fissure around the metallic artifacts.

Subarachnoid hemorrhage was not found on postprocedural MR imaging, which was performed after 110 minutes in 1 patient, 2 days in 2 patients, 3 days in 1 patient, 4 days in 2 patients, and 5 

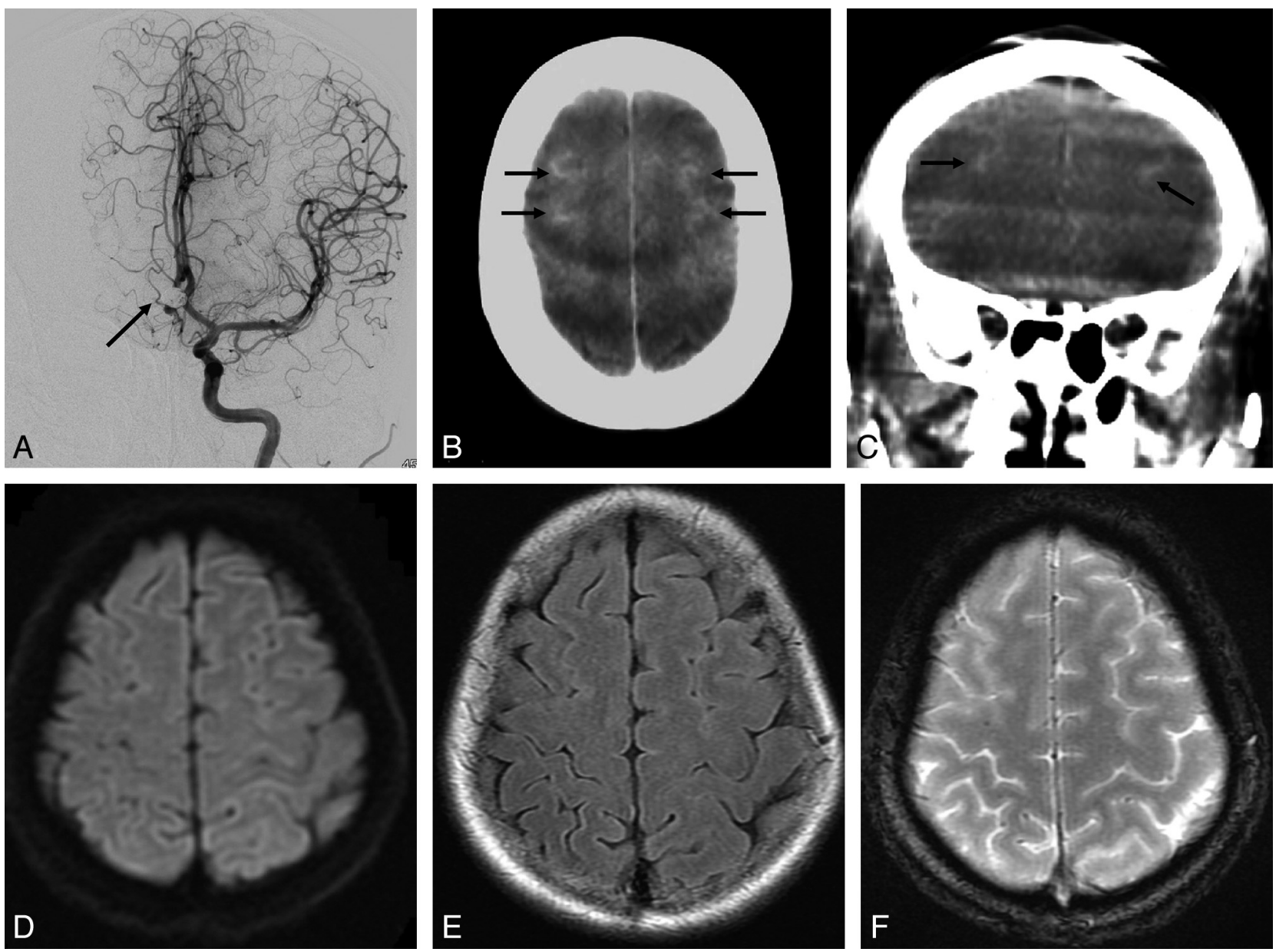

FIG 2. A 42-year-old woman with an unruptured 7-mm AcomA aneurysm. The patient underwent endovascular embolization for the aneurysm with the guiding catheter in the left ICA. The aneurysm was coiled with balloon assistance. The total volume of contrast medium injected was approximately $250 \mathrm{~mL}$. A, Postembolization DSA of the left ICA injection reveals coil mesh in the AcomA region (black arrow). Notice that the bilateral distal ACA is symmetrically visualized, presumably due to the hypoplastic right Al segment. $B$ and $C$, Axial and coronal CBCT scans, respectively, immediately after the procedure show focal subarachnoid hyperattenuation in the bilateral superior frontal sulci (black arrows). $D$, DWI reveals no abnormal high-intensity area indicating acute infarction. $E$, FLAIR imaging shows no high signal intensity in the sulci indicating $\mathrm{SAH} . F, \mathrm{~T} 2^{*}$-weighted imaging demonstrates no low signal intensity in the sulci indicating SAH. These MR images were obtained 2 days after the procedure.

days in 3 patients after coil embolization in these 9 patients. Statistically significant differences were observed between the presence of subarachnoid hyperattenuation and the total volume of contrast medium injected $(P<.001)$ and the size of the aneurysm $(P<.05)$. On the other hand, the location of the aneurysm, balloon inflation, and stent assistance were not significantly related to the presence of subarachnoid hyperattenuation $(P=.0728$, .567 , and .150 , respectively).

\section{DISCUSSION}

Endovascular coil embolization for unruptured intracranial aneurysms has been increasingly used in recent years. Although the safety and efficacy of this technique are now well-documented, intraprocedural complications such as aneurysmal rupture, thromboembolic events, and vasospasms can occur. ${ }^{4-6}$ It is important for the endovascular therapist to recognize and manage these serious complications as early as possible because a favorable clinical outcome can be expected if appropriate treatment is initiated within 30 minutes. ${ }^{14}$ From this point of view, CBCT immediately after embolization can be used to rule out unpredict- able intracranial complications without the need to transport the patient to a conventional CT facility.

Relatively few systematic evaluations of conventional CT findings after coil embolization for intracranial aneurysm have been reported. ${ }^{10-12}$ In a study, Baik et al ${ }^{11}$ found a focal subarachnoid hyperattenuation in 8 of 61 (13\%) conventional CT scans after uneventful endovascular treatment. Subarachnoid hyperattenuation may indicate transient contrast leakage into the subarachnoid space, resulting from temporary disruption of the bloodCSF interface by repeated contrast medium injection into a single vessel. $^{8-12}$

To the best of our knowledge, there are no reports describing subarachnoid hyperattenuation on postprocedural noncontrast CBCT. CBCT immediately after endovascular treatment in our study showed more frequent focal subarachnoid hyperattenuation (9 of 36 CBCT scans, $25.0 \%$ ) compared with that shown by conventional CT in the previous report. ${ }^{11}$ Ozturk et al ${ }^{10}$ described a significant inverse relationship between the occurrence of this finding and the elapsed time until the initial posttreatment con- 
ventional CT was performed; in other words, the more time elapsed, the less frequently those findings were observed. The result of our study is consistent with their description. Thus, the most likely reason for a high frequency of observed subarachnoid hyperattenuation is the very short interval between the end of the treatment and the CBCT scan, which was not enough time to wash out excessive iodine from the subarachnoid space. In addition, Baik et $\mathrm{al}^{11}$ reported that the attenuation of subarachnoid contrast leakage after embolization was higher than that of the usual SAH. Hyperattenuation of contrast medium itself may lead to the detectability of subarachnoid hyperattenuation on CBCT after embolization, though the contrast resolution of CBCT is considered to be inferior to that of conventional CT. $4,5,15$

In our study, CBCT showed subarachnoid hyperattenuation only at the superior frontal sulcus with or without involvement of the precentral sulcus. No previous study has performed a comprehensive investigation of the location of subarachnoid hyperattenuation, to our knowledge. Because all patients with subarachnoid hyperattenuation received aneurysm coil embolization in the anterior circulation, the contrast medium was mostly injected from the ICA and therefore may have tended to accumulate in the anterior watershed or ACA-MCA borderzone territory. Although the number of patients with an aneurysm in the posterior circulation in this study was small and focal subarachnoid hyperattenuation was not detected in the posterior circulation territory, focal subarachnoid hyperattenuation in the posterior watershed territory or posterior fossa should also be visible on postprocedural CBCT if the contrast medium is injected repeatedly from the vertebral artery. Thus, the specific location of subarachnoid hyperattenuation (eg, the watershed area within the relevant parent artery territory) may be an important clue for differentiation between benign contrast leakage and SAH.

Previous studies have described several patterns of postprocedural conventional CT findings, such as cortical, striatal, and intraventricular hyperattenuation as well as subarachnoid hyperattenuation, which showed either a single or a mixed pattern. In particular, cortical hyperattenuation was more frequent than the other findings. ${ }^{10-12}$ However, apparent cortical hyperattenuation in addition to subarachnoid hyperattenuation was not found on CBCT in this study. The image quality of CBCT, especially regarding the contrast resolution and generated image artifacts, may influence the visualization of the brain parenchyma, resulting in difficulty distinguishing cortical hyperattenuation due to contrast leakage from the normal attenuation of the cerebral cortex. ${ }^{5,15}$

In this study, we observed a statistical relationship between the amount of contrast medium injected and the occurrence of subarachnoid hyperattenuation, as previously reported. ${ }^{10-12}$ The bloodCSF or blood-brain interface, which is impermeable to contrast medium under normal conditions, can be injured by the hyperviscosity, hyperosmolarity, and chemotoxicity of the contrast medium when contrast is repeatedly injected into a single artery. ${ }^{8-12}$ However, a statistically significant relationship was not found between the use of balloon or stent assistance and the presence of subarachnoid hyperattenuation on CBCT. Ozturk et al ${ }^{10}$ reported that cortical hyperattenuation on postprocedural conventional CT is related to the number of inflations or total inflation time in procedures requiring balloon assistance, likely because vascular permeability increases due to temporary ischemia induced by frequent balloon inflation. No previous study has examined the relationship between stent assistance and the occurrence of subarachnoid hyperattenuation, to our knowledge. In this study, the stent-assisted technique did not always require increased injection of contrast medium and it did not always induce transient ischemia, which are factors related to subarachnoid contrast leakage. Further studies in larger groups of patients are needed to determine the effect of stent assistance and balloon assistance in this phenomenon.

One of the limitations of this study is that because of the retrospective study design, not all patients underwent immediate postprocedural conventional CT or MR imaging and no patients underwent lumbar CSF drainage after endovascular treatment to definitively exclude SAH. From our study, however, it seems that we can distinguish SAH from benign subarachnoid hyperattenuation by the location of subarachnoid hyperattenuation and the amount of contrast medium injected.

\section{CONCLUSIONS}

Subarachnoid hyperattenuation due to contrast leakage can be detected by noncontrast CBCT immediately after coil embolization for unruptured aneurysms. It is important to recognize subarachnoid hyperattenuation because it mimics $\mathrm{SAH}$, which requires significant immediate postprocedural medical management. Greater amounts of contrast medium given before CBCT and the specific location of subarachnoid hyperattenuation may help differentiate clinically benign focal subarachnoid hyperattenuation due to contrast leakage from SAH.

\section{ACKNOWLEDGMENTS}

We thank Naoki Iwata of the Division of Clinical Radiology, Tottori University Hospital, who provided support with regard to the technical terms related to the scanning system.

\section{REFERENCES}

1. Kalender W, Kyriakou Y. Flat-detector CT (FD-CT). Eur Radiol 2007;17:2767-79

2. Akpek S, Brunner T, Benndorf G, et al. Three-dimensional imaging and cone beam volume $\mathrm{CT}$ in $\mathrm{C}$-arm angiography with flat panel detector. Diagn Interv Radiol 2005;11:10-13

3. Heran NS, Song JK, Namba K, et al. The utility of DynaCT in neuroendovascular procedures. AJNR Am J Neuroradiol 2006;27:330-32

4. Doelken M, Struffert T, Richter G, et al. Flat-panel detector volumetric CT for visualization of subarachnoid hemorrhage and ventricles: preliminary results compared to conventional CT. $\mathrm{Neu}$ roradiology 2008;50:517-23

5. Struffert T, Richter G, Engelhorn T, et al. Visualization of intracerebral haemorrhage with flat-detector CT compared to multislice CT: results in 44 cases. Eur Radiol 2009;19:619-25

6. Engelhorn T, Struffert T, Richter G, et al. Flat-panel detector angiographic $\mathrm{CT}$ in the management of aneurysmal rupture during coil embolization. AJNR Am J Neuroradiol 2008;29:1581-84

7. Struffert T, Deuerling-Zheng Y, Kloska S, et al. Flat detector CT in the evaluation of brain parenchyma, intracranial vasculature, and cerebral blood volume: A pilot study in patients with acute symptoms of cerebral ischemia. AJNR Am J Neuroradiol 2010;31:1462-69

8. Numaguchi Y, Fleming MS, Hasuo K, et al. Blood-brain barrier disruption due to cerebral arteriography: CT findings. J Comput Assist Tomogr 1984;8:936-39

9. Uchiyama Y, Abe T, Hirohata M, et al. Blood-brain barrier disrup- 
tion of nonionic iodinated contrast medium following coil embolization of a ruptured intracerebral aneurysm. AJNR Am J Neuroradiol 2004;25:1783-86

10. Ozturk A, Saatci I, Pamuk AG, et al. Focal increased cortical density in immediate postembolization CT scans of patients with intracranial aneurysms. AJNR Am J Neuroradiol 2006;27:1866-75

11. Baik SK, Kim YS, Lee HJ, et al. Immediate CT findings following embolization of cerebral aneurysms: suggestion of blood-brain barrier or vascular permeability change. Neuroradiology 2008;50: 259-66

12. Brisman JL, Jilani M, McKinney JS. Contrast enhancement hyper- density after endovascular coiling of intracranial aneurysms. AJNR Am J Neuroradiol 2008;29:588-93

13. Söderman M, Babic D, Holmin S, et al. Brain imaging with a flat detector C-arm. Neuroradiology 2008;50:863-68

14. Carvi y Nievas M, Haas E, Höllerhage HG. Severe intracranial bleedings during endovascular procedures: outcome of surgically treated patients. Neurol Res 2007;29:81-90

15. Orth RC, Wallace MJ, Kuo MD. Technology Assessment Committee of the Society of Interventional Radiology: C-arm cone-beam CTgeneral considerations for use in interventional radiology. $J$ Vasc Interv Radiol 2008;19:814-20 